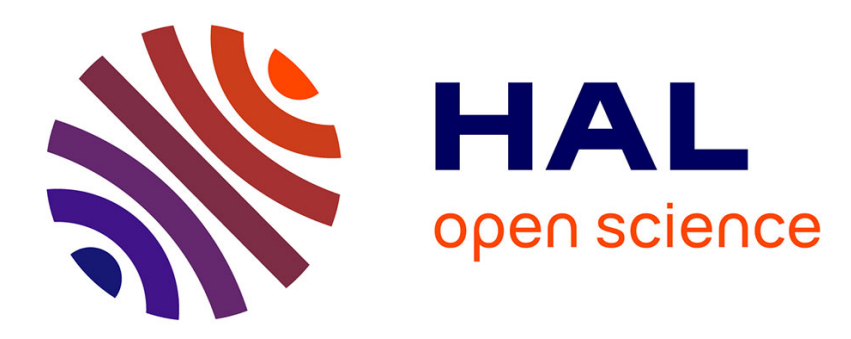

\title{
On the cross-over from percolation to conduction in a random potential
}

\author{
J. Friedel
}

\section{To cite this version:}

J. Friedel. On the cross-over from percolation to conduction in a random potential. Journal de Physique Lettres, 1976, 37 (1), pp.9-11. 10.1051/jphyslet:019760037010900 . jpa-00231226

\section{HAL Id: jpa-00231226 https://hal.science/jpa-00231226}

Submitted on 1 Jan 1976

HAL is a multi-disciplinary open access archive for the deposit and dissemination of scientific research documents, whether they are published or not. The documents may come from teaching and research institutions in France or abroad, or from public or private research centers.
L'archive ouverte pluridisciplinaire HAL, est destinée au dépôt et à la diffusion de documents scientifiques de niveau recherche, publiés ou non, émanant des établissements d'enseignement et de recherche français ou étrangers, des laboratoires publics ou privés. 


\title{
ON THE CROSS-OVER FROM PERCOLATION TO CONDUCTION IN A RANDOM POTENTIAL
}

\author{
J. FRIEDEL \\ Physique des Solides (*), Université Paris-Sud Orsay, France
}

(Reçu le 16 septembre 1975, accepté le 23 octobre 1975)

\begin{abstract}
Résumé. - La proposition récente de Toulouse pour l'énergie de transition entre percolation classique et conduction quantique dans un potentiel aléatoire est obtenue en comparant la profondeur de pénétration tunnel à la taille des régions permises classiquement.
\end{abstract}

\begin{abstract}
Toulouse's recent proposal for cross-over energy between classical percolation and quantum conduction in a random potential is obtained by comparing the tunnelling depth to the size of the classically allowed regions.
\end{abstract}

Let $E_{\mathrm{p}}$ be the classical percolation energy for a given random potential. Because of possible tunnel effects, quantum particles can produce a conductive state down to an energy $E_{\mathrm{c}}$ which differs from $E_{\mathrm{p}}$. At least, in two or three dimensions, it is actually below $E_{\mathrm{p}}$.

The cross-over energy $E^{*}$ of the problem is the energy below which these quantum effects become noticeable.

To energy $E^{*}$, Toulouse [1] associates an energy $E^{* *}$ such that

$$
\Delta E=E^{*}-E_{\mathrm{p}}=E_{\mathrm{p}}-E^{* *} .
$$

He proposes to define $E^{* *}$ as the quantized energy nearest to $E_{\mathrm{p}}$ in the classically localized regions, of average size $\xi$ :

$$
\Delta E \simeq\left(\rho_{E} \xi^{d}\right)^{-1}
$$

$\rho_{E}$ is here the density of states per unit energy and unit volume near $E_{\mathrm{p}}$, and $d$ the dimensionality of the problem; use is made of the fact that, near $E_{\mathrm{p}}$, the allowed regions occupy a finite fraction of the whole volume. As, for $\Delta E \rightarrow 0$,

$$
\xi \simeq a(\Delta E)^{-v}
$$

where $a$ is a constant which only depends on the spatial variations of the potential, Toulouse deduces

$$
\frac{\xi}{a} \simeq\left(a^{d} \rho_{E}\right)^{\frac{v}{1-v d}}
$$

$\left(^{*}\right)$ Laboratoire associé au C.N.R.S. and

$$
\Delta E \simeq\left(a^{d} \rho_{E}\right)^{\frac{1}{v d-1}} .
$$

These formulae have power laws in $\rho_{E}$ which look attractive. However, as $a$ is a purely classical constant related to the percolation potential, the quantum nature of the conduction problem only appears in the value of the density of states $\rho_{E}$; also criterion (2) looks somewhat arbitrary and is not clearly related to a tunnel effect.

- In this note, we point out that relation (5) can also be obtained by stating that, at the critical energy $E^{* *}$, the average tunnelling depth around the allowed regions is of the same order of magnitude as the average size of the classically allowed regions.

From relation (3), we deduce that near $E_{\mathrm{p}}$ the potential near the border of each allowed region has an average radial variation

$$
\bar{V}(2 r) \simeq E_{\mathrm{p}}-\left(\frac{2 r}{a}\right)^{-\frac{1}{v}}
$$

where $r$ is the radius counted from the center of the allowed region. For $E \rightarrow E_{\mathrm{p}}$

$$
\bar{V}^{\prime}(2 r) \simeq \frac{1}{v a}\left(\frac{2 r}{a}\right)^{-\left(\frac{1}{v}+1\right)}
$$

tends to zero near the border of the allowed regions $(2 r \simeq \xi \rightarrow \infty)$. A WKB approximation thus holds to compute both the size $\delta \xi$ of the tunnelling depth and the density of states $\rho_{E}$. 
1. In the tunnelling region $2 r>\xi$, the wave Hence function of energy

$$
E=E_{\mathrm{p}}-\left(\frac{\xi}{a}\right)^{-\frac{1}{v}}=\bar{V}(\xi)
$$

$$
-\int_{r}^{\frac{1}{2} \xi} k \mathrm{~d} r \simeq\left[\frac{m}{\hbar^{2}} \bar{V}^{\prime}(\xi)\right]^{1 / 2}(\xi-\dot{2} r)^{3 / 2} .
$$

decreases exponentially with distance as

$$
\exp -\int_{\frac{1}{2} \xi}^{r} K \mathrm{~d} r
$$

where

$$
K^{2}=\frac{m}{\hbar^{2}}[\bar{V}(2 r)-\bar{V}(\xi)] \simeq \frac{m}{\hbar^{2}} \bar{V}^{\prime}(\xi)(2 r-\xi) .
$$

Hence an exponential decrease as

$$
\left[\frac{m}{\hbar^{2}} \bar{V}^{\prime}(\xi)\right]^{1 / 2}(2 r-\xi)^{3 / 2}
$$

and a tunnelling depth

$$
\delta \xi \simeq\left[\frac{\hbar^{2}}{m \bar{V}^{\prime}(\xi)}\right]^{1 / 3}
$$

or, using (7) :

$$
\left(\frac{\delta \xi}{a}\right)^{3} \simeq\left(\frac{\hbar^{2} v}{m a^{2}}\right)\left(\frac{\xi}{a}\right)^{\frac{1}{v}+1}
$$

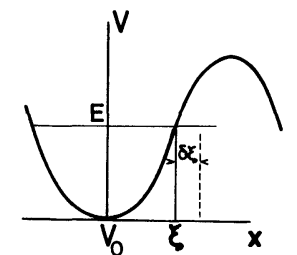

a

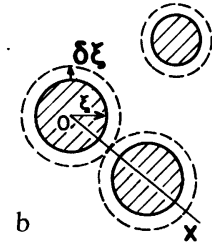

FIG. 1. - Definition of $\xi$ and $\delta \xi: \mathrm{a}:$ in energy; b : in space.

If we then require

$$
\delta \xi=\xi
$$

we obtain

$$
\frac{\xi}{a}=\left(\frac{\hbar^{2} v}{m a^{2}}\right)^{\frac{1}{2-(1 / v)}} .
$$

2. In the allowed region $(2 r<\xi)$, the wave function oscillates as

where

$$
\sin \left(\text { const. }-\int_{r}^{\frac{1}{2} \xi} k \mathrm{~d} r\right)
$$

$$
\begin{aligned}
& k^{2}=-K^{2}=\frac{m}{\hbar^{2}}[\bar{V}(\xi)-\bar{V}(2 r)] \simeq \\
& \simeq \frac{m}{\hbar^{2}} \bar{V}^{\prime}(\xi)(\xi-2 r) .
\end{aligned}
$$

A change $\mathrm{d} E$ in energy introduces a phase shift

$$
\mathrm{d} \delta=\frac{\mathrm{d}}{\mathrm{d} \xi}\left(-\int_{r}^{\frac{1}{2} \xi} k \mathrm{~d} r\right) \frac{\mathrm{d} \xi}{\mathrm{d} E} \mathrm{~d} E
$$

which, for $2 r \ll \xi$, and using (7), is of order

$$
\mathrm{d} \delta \simeq\left(\frac{m}{\hbar^{2}}\right)^{1 / 2} a\left(\frac{\xi}{a}\right)^{\frac{1}{2 v}+1} \mathrm{~d} E .
$$

The derivative of $\delta$ with energy gives, to a factor $\pi$, the density of states $\rho_{E} \xi^{d}$ in one allowed region :

$$
\rho_{E} \xi^{d} \simeq\left(\frac{m}{\hbar^{2}}\right)^{1 / 2} a\left(\frac{\xi}{a}\right)^{\frac{1}{2 v}+1} .
$$

The use of eq. (12), (14) then lead to Toulouse's eq. (4) and (5).

Discussion. - 1. Eq. (14) can also be obtained by computing the density of states for a harmonic oscillator of classical size $\xi$ and of potential slope $\bar{V}^{\prime}(\xi)$ given by (7) at its classical size :

$$
\rho_{E} \xi^{d} \simeq \frac{n^{d-1}}{\hbar \omega}=\frac{(n \hbar \omega)^{d-1}}{(\hbar \omega)^{d}}
$$

with

$$
\begin{aligned}
\hbar \omega & =\left(\frac{\hbar^{2} A}{m}\right)^{1 / 2} \\
n \hbar \omega & =A \xi^{2} \\
\bar{V}^{\prime}(\xi) & \simeq A \xi .
\end{aligned}
$$

One obtains :

$$
\rho_{E} \xi^{d} \simeq\left(\frac{m}{\hbar^{2}}\right)^{\frac{d}{2}} A^{\frac{d}{2}-1} \xi^{2(d-1)}
$$

with

$$
A=\frac{1}{v}\left(\frac{\xi}{a}\right)^{-\left(\frac{1}{v}+2\right)} a^{-2} .
$$

Hence eq. (14).

2. Eq. (18) clearly shows that the effective harmonic oscillator potential varies with the size $\xi$ of the allowed regions.

If, on the contrary, one assumed its curvature $A$ to be independent of $\xi$, eq. (9), (15) and (16) would lead to

$$
\delta \xi \simeq\left(\frac{\hbar^{2}}{m A \xi}\right)^{1 / 3}
$$


Thus, $\delta \xi \simeq \xi$ if

$$
\xi \simeq\left(\frac{\hbar^{2}}{m A}\right)^{1 / 4} \simeq\left(\frac{\hbar^{2}}{m A \xi^{2}}\right)^{1 / 2}
$$

i.e. for $\xi$ of the order of the wave length in the allowed region. Putting (19) into (17) would give

and

$$
\xi \simeq\left(\frac{\hbar^{2} \rho_{E}}{m}\right)^{\frac{1}{2-d}}
$$

$$
\Delta E \simeq\left(\frac{\hbar^{2} \rho_{E}}{m}\right)^{\frac{1}{v(d-2)}}
$$

This is not very different from Toulouse's relations (4) and (5), except for the replacement of $v$ by its classical value $\frac{1}{2}$ in part of the exponent.

Eq. (20), however, does not take such good account of the actual average potential in and near the allowed regions, and is therefore likely to be less acceptable. Besides random short range fluctuations which are averaged out in both models, the potential $V$ is expected to have a well defined minimum inside each allowed region, bordered in most directions by well defined maxima well above the percolation energy $E_{\mathrm{p}}$; only in some directions, neighbouring allowed regions connect through passes with maximum energy near to $E_{\mathrm{p}}$. The model of this paragraph, leading to eq. (20), essentially neglects those special low energy directions around an allowed region; the model used in the main part of this note, and leading to eq. (4), averages out $V$ in all directions, taking a weighted account of those special low energy directions. This model of eq. (4) looks more reasonnable, but should in all rigour be tested by studying the possible effect of anisotropy in the radial variation of the potential around an allowed region.

3. For a given energy $E$, thus a given size $\xi$, eq. (10) shows that the tunnelling depth $\delta \xi$ tends to zero with $\hbar^{2} / m$, as it should, i.e. when quantum effects become negligible.

Eq. (3) and (12) show however that, as $v>\frac{1}{2}$, the critical size $\xi$ tends to zero in the classical limit $\hbar^{2} / m \rightarrow 0 ; \Delta E$ tends to infinity, and a cross-over energy $E^{*}$, as defined by eq. (1), has clearly no physical meaning in this limit.

4. As $\frac{1}{2}<v<1$, eq. (5) shows that the exponent $1 / v d-1$ has different signs for $d=1$ on the one hand, and $d \geqslant 2$ on the other. This might be related to the fact that the cross-over energy $E^{*}$ is infinite for $d=1$ : all quantum states are non conducting, while percolation occurs above a well defined energy limit $E_{\mathrm{p}}$. It seems however that the absence of conductivity for $d=1$ cannot be explained without a deeper analysis of the quantum states.

These two last remarks cast some doubt on the generality of relations (1) and (11).

\section{References}

[1] Toulouse, G., C. R. Hebd. Séan. Acad. Sci. 280B (1975) 629. 